\title{
Coherent motion of chaotic attractors
}

\author{
Patrick Louodop, ${ }^{1,2}$ Suman Saha, ${ }^{3,4}$ Robert Tchitnga, ${ }^{2,5}$ Paulsamy Muruganandam ${ }^{6}$ Syamal K. Dana, ${ }^{7,8}$ and Hilda A. Cerdeira ${ }^{1}$ \\ ${ }^{1}$ São Paulo State University (UNESP), Instituto de Física Teórica, Rua Dr. Bento Teobaldo Ferraz 271, Bloco II, \\ Barra Funda, 01140-070 São Paulo, Brazil \\ ${ }^{2}$ Laboratory of Electronics and Signal Processing, Faculty of Science, Department of Physics, University of Dschang, \\ P.O. Box 67 Dschang, Cameroon \\ ${ }^{3}$ Dumkal Institute of Engineering and Technology, Murshidabad 742406, India \\ ${ }^{4}$ Department of Instrumentation and Electronics Engineering, Jadavpur University, Kolkata 700090, India \\ ${ }^{5}$ Institute of Surface Chemistry and Catalysis, University of Ulm, Albert-Einstein-Allee 47, 89081 Ulm, Germany \\ ${ }^{6}$ Department of Physics, Bharathidasan University, Tiruchirapalli 620024, Tamil Nadu, India \\ ${ }^{7}$ Department of Mathematics, Jadavpur University, Kolkata 700032, India \\ ${ }^{8}$ Centre for Complex System Research Kolkata, Kolkata 700094, India
}

(Received 9 February 2017; revised manuscript received 10 August 2017; published 18 October 2017)

\begin{abstract}
We report a simple model of two drive-response-type coupled chaotic oscillators, where the response system copies the nonlinearity of the driver system. It leads to a coherent motion of the trajectories of the coupled systems that establishes a constant separating distance in time between the driver and the response attractors, and their distance depends upon the initial state. The coupled system responds to external obstacles, modeled by short-duration pulses acting either on the driver or the response system, by a coherent shifting of the distance, and it is able to readjust their distance as and when necessary via mutual exchange of feedback information. We confirm these behaviors with examples of a jerk system, the paradigmatic Rössler system, a tunnel diode system and a Josephson junction-based jerk system, analytically, to an extent, and mostly numerically.
\end{abstract}

DOI: 10.1103/PhysRevE.96.042210

\section{INTRODUCTION}

Studies on synchronization of dynamical systems started with Christiaan Huygens in 1665 [1] and, in the past three decades, have become a subject of intensive research with prospective applications in practical systems [2] mainly due to the confirmation of the existence of synchronization in chaotic systems $[3,4]$. Although the technological prospect is not promising [5], so far, studies on synchronization are able to explain many natural behaviors [3,6,7]. In the past 15 years, the synchronization studies have been extended from a small ensemble of dynamical systems to a large network of oscillators [3]. The current focus is on investigations of complex networks [8-10], small world and scale-free networks, and additionally, attributing dynamics to individual nodes of a network.

A related issue of concern is how an ensemble of dynamical units organizes a collective motion. How robust is this selforganized behavior against external perturbation? Is there any internal mechanism of restoring a desired state of activity? An interesting example of a collective activity in motion is the flocking of birds [11-13], which has shown collective decision-making during their flights in a coherent manner. A leader-follower-type cohesive motion prevails in the flocking of starlings, swimming fishes, where it is important that the follower copies [14-16] the decision-making process of the leader. The collective motion of such living objects is difficult to understand and usually explained using the concept of statistical mechanics. A dynamical system approach may provide additional clues to unravel the mystery of such cohesive motion. We investigate a simple dynamical model of two drive-response-type coupled systems that creates a coherent leader-follower dynamics in state space. In this context, we apply a type of unidirectional interaction as typically used for leader-follower coherent dynamics. It is well known $[1,3,4]$ that, using a simple diffusive coupling, one can establish coherent dynamics of different forms, complete synchronization (CS), phase synchronization (PS), or generalized synchronization (GS) in chaotic systems $[17,18]$. Pecora and Carroll [4] defined the coupling as diffusive, scalar type, using single variable separately when the coupling threshold for synchronization differs for each type. Their goal was to achieve complete synchronization. In our case the target is to realize a coherent motion between two chaotic attractors, but with a distance of separation between them. To achieve it, we derive the coupling functions using the Lyapunov function stability. To obtain this goal we make a slightly different proposition that the response dynamical system or the follower makes a decision of copying the nonlinearity function of the driver system that establishes a type of unidirectional coupling interaction with the driver. A situation eventually emerges when the response attractor certainly realizes a type of coherent dynamics, however, atypically maintains a constant distance with the driver. More categorically, in the dynamical sense, a subgroup of response variables has identical amplitude and phase with their corresponding driver variables but preserves a constant distance, while the rest of the pairs of driver-response variables keep identical amplitude and phase with no distance of separation in time. As a whole, it reflects a scenario where the trajectories of a pair of leader-follower attractors establish a form of coherent motion with a constant distance of separation in state space. The separating distance is decided by the initial distance where they start from. This leads us to draw an analogy with the coherent motion of two flying birds following each other in a leader-follower strategy but keeping a safe distance. The analogy seems more realistic when we study the response of the coherent oscillators, both the leader and follower, to external pulses. 
We first present a simple two-coupled model of a jerk system to illustrate the coherent dynamics with a leaderfollower strategy. Next, we model a situation to find the reaction of this coherent dynamics to an external disturbance by applying an external impulse. The response attractor moves away from the driver in response to the external impulse; it creates an additional distance further away from the original distance with the driver that depends upon the height and duration of the external impulse. The response system can restore its original position when a negative pulse is applied or an appropriate feedback pulse is sent to the driver. This overall coherent dynamics is not restricted to the jerk system only; we have confirmed the phenomenon in the Rössler system as well as the tunnel diode systems and Josephson junctions based jerk system, where we use the same strategy of appropriately copying the nonlinearity of the driver into the response system, as shown in the Appendices A, B, and C.

Summarizing, we introduce the concept of synchronization as a coherent motion of the trajectories of a pair of leaderfollower dynamical systems and their displacement, their responses to external disturbances, and a possible technique of how they restore their original positions in state space.

\section{COHERENT DYNAMICS: DISPLACEMENT OF COUPLED SYSTEMS}

We consider two unidirectionally coupled jerk systems, ${ }^{1}$ where the nonlinearity appears in a piecewise linear form. The driver system, $X=\left[x_{1}, x_{2}, x_{3}\right]$, is

$$
\begin{aligned}
& \dot{x}_{1}=I\left(x_{2}\right)+\epsilon_{1} \delta_{M}(\tau), \quad \dot{x}_{2}=\alpha\left[-x_{3}+I\left(x_{2}\right)\right], \\
& \dot{x}_{3}=\beta\left(-x_{1}+x_{2}-\gamma x_{3}\right),
\end{aligned}
$$

and the response system, $Y=\left[y_{1}, y_{2}, y_{3}\right]$, is

$$
\begin{aligned}
& \dot{y}_{1}=I\left(x_{2}\right)+\epsilon_{2} \delta_{S}(\tau), \quad \dot{y}_{2}=\alpha\left[-y_{3}+I\left(x_{2}\right)\right], \\
& \dot{y}_{3}=\beta\left(-y_{1}+y_{2}-\gamma y_{3}\right),
\end{aligned}
$$

where the piecewise linear function is

$$
I\left(x_{2}\right)=\left\{\begin{array}{cl}
-x_{2}, & \text { if } x_{2} \leqslant 1, \\
-1, & \text { otherwise, }
\end{array}\right.
$$

and

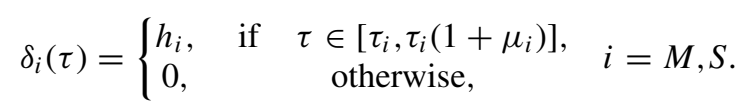

$\epsilon_{1,2}=1$ corresponds to the application of impulses of height $h_{i}$ and duration $\mu_{i}$ to both the driver and the response oscillators, and $\epsilon_{1,2}=0$ for no external impulse. The piecewise linear term $I\left(x_{2}\right)$ of the driver is copied in the response system and basically, a unidirectional coupling is thereby established

\footnotetext{
${ }^{1}$ A system is called jerk when the flow can be rewritten as a third order differential equation in a single scalar variable [19,24,25], When $\epsilon_{1,2}=0$, we can rewrite Eq. (1) as:

$$
\dddot{x}_{2}=\alpha\left(-\beta\left(-\dot{x}_{1}+\dot{x}_{2}-\gamma \ddot{x}_{1}+\frac{\gamma}{\alpha} \ddot{x}_{2}\right)+\dddot{x}_{1}\right) .
$$

where $\ddot{x}_{1}=I^{\prime}\left(x_{2}\right) \dot{x}_{2}$ and $\dddot{x}_{1}=I^{\prime \prime}\left(x_{2}\right) \dot{x}_{2}^{2}+I^{\prime}\left(x_{2}\right) \ddot{x}_{2} . \dddot{x}_{2}$ is called jerk function.
}

between the two jerk systems. The error dynamics is defined by $e_{i}=x_{i}-y_{i}$,

$$
\dot{e}_{1}=0, \quad \dot{e}_{2}=-\alpha e_{3}, \quad \dot{e}_{3}=\beta\left(-e_{1}+e_{2}-\gamma e_{3}\right) .
$$

Based on the following selected Lyapunov function,

$$
V=\frac{1}{2}\left(e_{1}^{2}+\frac{1}{\alpha} e_{2}^{2}+\frac{1}{\beta} e_{3}^{2}\right)
$$

it is established (assuming $\alpha>0, \beta>0$ and $\gamma>0$ ) that the system defined by Eq. (5) is practically stable [20] since the time derivative of the Lyapunov function in Eq. (6) is bounded by a positive constant. This also means that the error between the driver and response systems is sufficiently small, but different from zero and could be considered as a tolerance in the synchronization condition $[21,22]$ :

$$
\dot{V} \leqslant \frac{e_{1}^{2}}{4 \gamma}
$$

This boundedness is ensured by the fact that $e_{1}(\tau)$ is constant [see Eq. (5)]. A type of GS thus emerges between the driver and response when two pairs of state variables of the coupled system are identical in phase and amplitude but maintain a constant distance of separation (say, $d$, when $x_{1}=y_{1}+d_{1}$, $x_{2}=y_{2}+d_{2}$ ) while the third pair of variables is in a state of CS of amplitude and phase with zero distance $\left(x_{3}=y_{3}\right)$. We have verified numerically that the constant distance of separation and the copy of the nonlinearity of the driver by the response are necessary conditions to obtain the emergent GS. We also notice that the jerk system is invariant under the transformation $x_{1}->x_{1}+d, x_{2}->x_{2}+d$, but this is not the case in the other systems studied (see Appendices).

For numerical confirmation, we choose a set of parameters, $\alpha=0.025, \beta=0.765, \gamma=0.0938$ such that the stability condition $\left(\beta=4 \alpha / \gamma^{2}\right)$ is satisfied; the initial conditions are chosen as $\left(0.1,-5 \times 10^{-7},-1\right)$ for the driver system and $(0.7,0.5,0.7)$ for the response system. We notice a displacement of the response attractor from the driver attractor that is guided by the initial conditions. For a visual demonstration, we fix the initial conditions of the driver and change the initial conditions of the response system; the driver and the response systems maintain GS, as explained above, but change the distance of separation with varying initial conditions as shown in state space in Fig. 1 . We reset $x_{1}(0)=0$ and plot the driver attractor (blue) as well as the response attractor for two different initial conditions, (a) $y_{1}(0)=40$ (green), (b) $y_{1}(0)=-40$ (red), in Figs. 1(a) and 1(b), respectively. The response attractor thus moves away from the driver for a change of one initial condition only in $e_{1}(0)=x_{1}(0)-y_{1}(0)$, while the other initial condition is kept unchanged. To quantify the displacement, in other words, the distance of separation between the two systems, we define a measure,

$$
d=\operatorname{sgn} y_{1}(0) \times \sqrt{\sum_{i=1}^{3}\left(x_{i}-y_{i}\right)^{2}} .
$$

The distance $d$ of the response attractor from the driver attractor, for various choices of $y_{1}(0)$, is plotted in Fig. 1(c). In all the cases, the distance $d$ is stabilized in time to a constant 
(a)
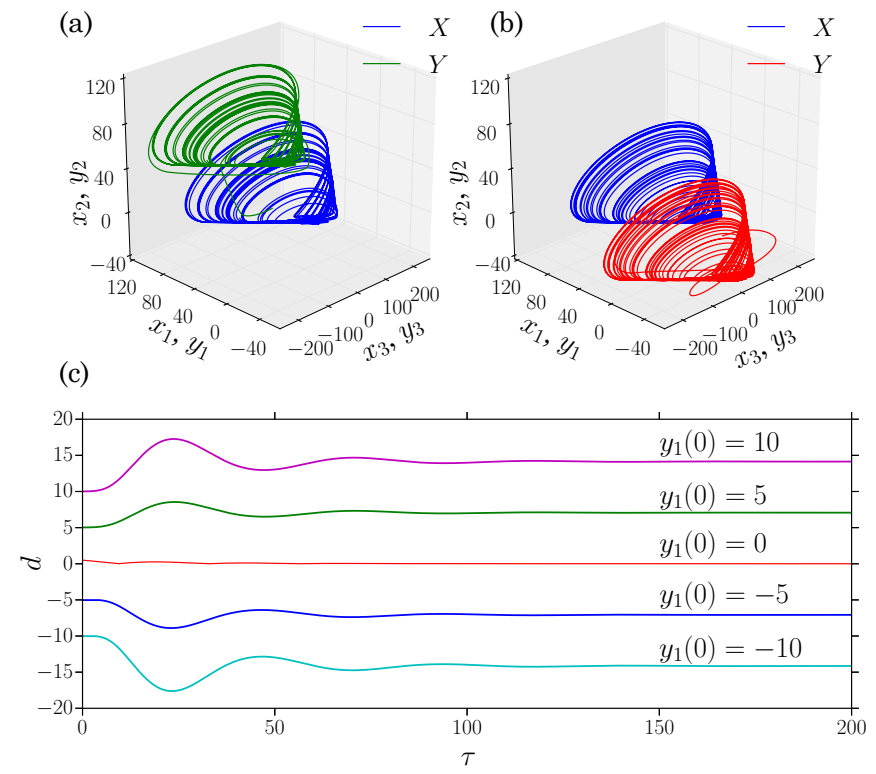

FIG. 1. Displacement of response attractors for (a) $y_{1}(0)=40$ (green), (b) $y_{1}(0)=-40$ (red) from the driver (blue), (c) plot of the distance between drive (blue) and response attractors for different initial conditions of $y_{1}$ for fixed set of $\left\{x_{1}(0), x_{2}(0), x_{3}(0), y_{2}(0), y_{3}(0)\right\}=$ $\left\{0,-5 \times 10^{-7},-1,0.5,0.7\right\}$. Other parameters: $\alpha=0.025, \beta=$ $0.765, \gamma=0.0938, \epsilon_{1,2}=0$.

value after initial small oscillations as decided by different choices of initial conditions.

\section{A. Displacement of response attractor: External impulse}

We find a very interesting consequence of this nontrivial leader-follower coherent dynamics with a distance of separation between the two attractors. We apply an external impulse in the form of a square pulse to the response system by setting $\epsilon_{1}=0$ and $\epsilon_{2}=1$. It appears from Fig. 2(a) that the response variable $y_{1}$ (red line) jumps to a higher level at the start of the impulse (green). As already established by Eqs. (5) and (7) that $e_{3}(t) \rightarrow 0$ and it implies $e_{1}=e_{2}(t) \rightarrow \zeta$ and equal to the initial difference $e_{1}(0)=x_{1}(0)-y_{1}(0)$. Obviously, the response variable $y_{2}$ faces a similar kicking (not shown here) while $y_{3}$ remains unchanged since it always maintains CS with $x_{3}$. This asserts that the $y_{3}$ variable, despite receiving an initial kick, restores its original position quickly (after a small transient), as shown in Fig. 2(b), while $y_{1}\left(y_{2}\right)$ continues to maintain the distance $d_{1}\left(d_{2}\right)$ with $x_{1}\left(x_{2}\right)$. Eventually, the driver and response attractors establish a new distance of separation and maintain it for the long run as shown in Fig. 2(c); they never lose their coherent dynamics. The additional distance of separation in response to the external impulse has a finite value that depends upon $h_{i}$ and $\mu_{i}$ of the pulse.

We mention here that, for simplicity, we use a set of initial conditions so that $e_{1}=x_{1}(0)-y_{1}(0)=0$ before applying the pulse, and hence the drive-response system has zero distance of separation in the beginning. A separating distance $d_{1}\left(d_{2}\right)$ is created between $x_{1}$ and $y_{1}\left(x_{2}\right.$ and $\left.y_{2}\right)$ by the impact of the pulse and this distance varies linearly with the height $h$ of an impulse for a fixed $\mu$ (not shown here). On the other
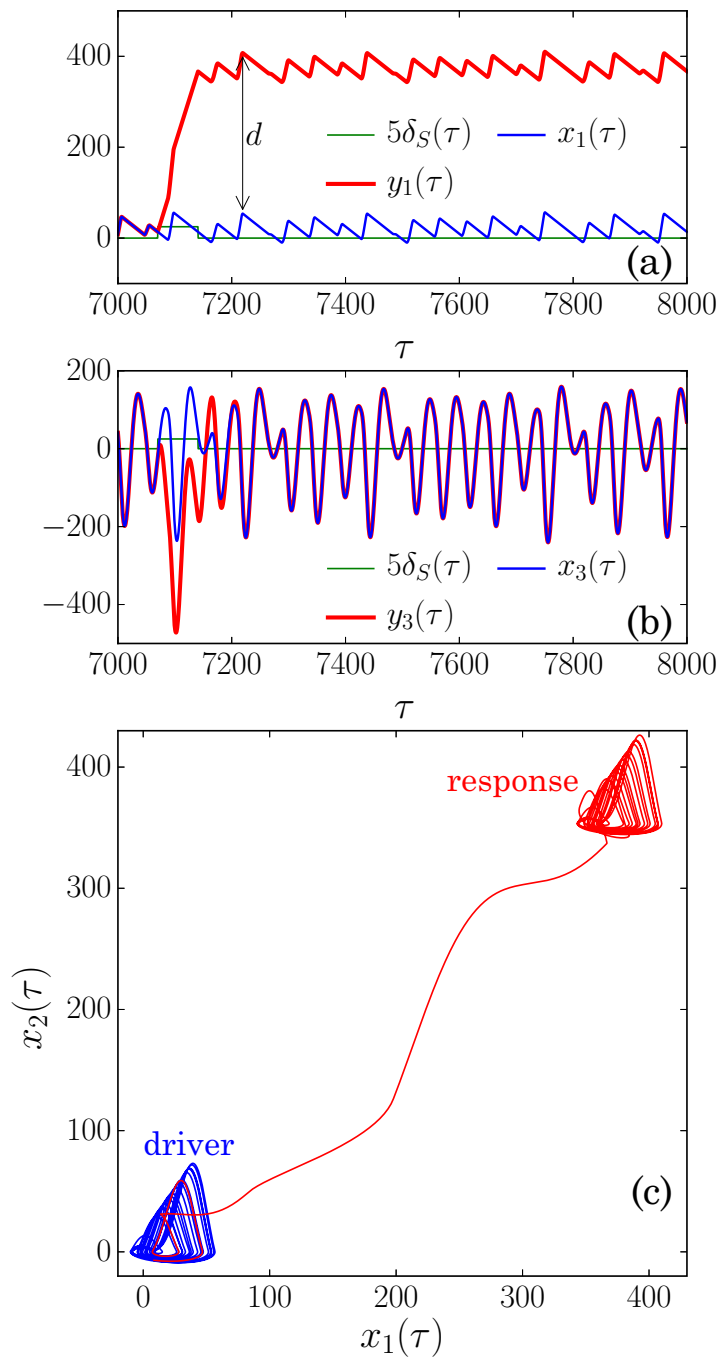

FIG. 2. Displacement of the response attractor by external impulse. Upper panel shows the driver variable (blue line) $x_{1}$, response variable (red line) $y_{1}$, and the external short pulse (green line). Middle panel shows driver $\left(x_{3}\right)$ and response variables $\left(y_{3}\right)$. Lower panel shows that the response attractor (red line) moves further away from the driver (blue).

hand, when varying the width of the pulse with a fixed $h$, we obtain three different kinds of behaviors (regions I, II, and III) as shown in Fig. 3(a). It clearly reflects that, for a reasonably large range of $\mu$ (fixed $h$ ), the impulse has no influence on $d_{1}$, which follows its initial value $d_{1}=e_{1}=x_{1}(0)-y_{1}(0)$ and then exponentially increases and finally saturates at a constant value for large $\mu$. Note that $d_{1}$ appears to be 0 in Fig. 3(a) in the lower range of $\mu$. An enlarged view of regions I and II in Fig. 3(b), in the lower range of $\mu$, reveals the actual scenario. The distance $d_{1}$ remains constant with $\mu$ and then, to our surprise, it becomes exponentially zero at a critical value near $\mu=-8$ (in log scale) (region II) where CS is established between the attractors (no distance of separation) before it starts increasing exponentially once again with the further increase of $\mu$ [region II, Figs. 3(a) and 3(b)]

The most interesting behavior appears in region II: instead of changing $y_{1}(0)$ if $x_{1}(0)$ (an initial value of the driver) is 

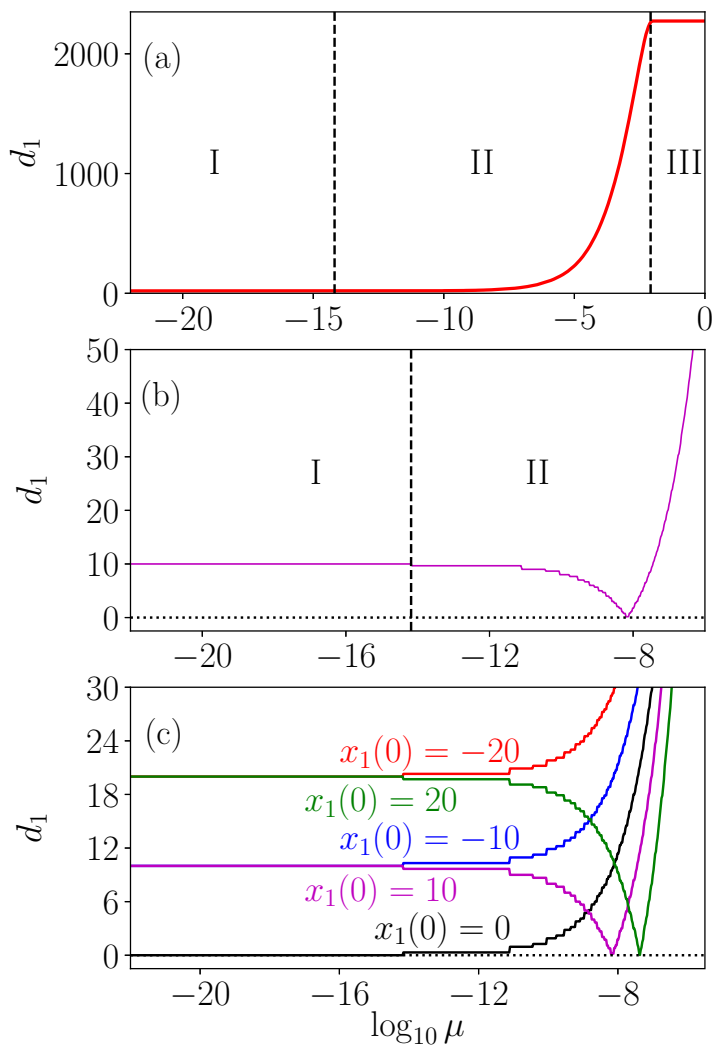

FIG. 3. Separating distance of the drive-response attractors against the width $\mu$ of the external pulse for $x_{1}(0)=10$ and $y_{1}(0)=0$. Panel (a) and its zoom, panel (b), show the stable distance $d_{1}=$ $x_{1}-y_{1}$ in region I and its nonlinear behavior in region II. Panel (c) shows that the nonlinear behavior of $d_{1}$ in region II depends strongly on the sign of $x_{1}(0)$ since $y_{1}(0)=0$. We found that this depends on the Lyapunov function, as explained in the text.

varied, $d_{1}$ is found sensitive to the sign and absolute value of $x_{1}(0)$. Figure 3(c) shows that, for $x_{1}(0)>0$, a critical value of $\mu$ exists, such that for any set of $\left(x_{1}(0), y_{1}(0)\right)$, the coupled system achieves a CS state when $d_{1} \simeq 0$. In contrast, for negative values of $x_{1}(0)<0$, the attractors never come close rather their distance of separation increases exponentially in the large limit of $\mu$. An important point to note that, in all the cases, in the lower limit and for a reasonably large range of $\mu$, this variation has no effect on the response system. Considering the impulse in the coupled system Eqs. (1)-(4), the new error dynamics is written as

$$
\dot{e}_{1}=-\delta(\tau), \quad \dot{e}_{2}=-\alpha e_{3}, \dot{e}_{3}=\beta\left(-e_{1}+e_{2}-\gamma e_{3}\right),
$$

and the time derivative of the Lyapunov function in Eq. (6) is given by

$$
\dot{V}_{1} \leqslant \frac{e_{1}^{2}}{4 \gamma}-\delta(\tau) e_{1} .
$$

Without the impulse, $e_{1}(\tau)$ is a constant and equal to $e_{1}(0)$, thus one can conclude that the impulse introduces an amount of energy to the response system, which could be assumed proportional to the area of the impulse $E_{\mathrm{imp}}=e_{1}(0) h \mu$. Accordingly, if $x_{1}(0)<0$, it implies $e_{1}(0)<0$ and the time
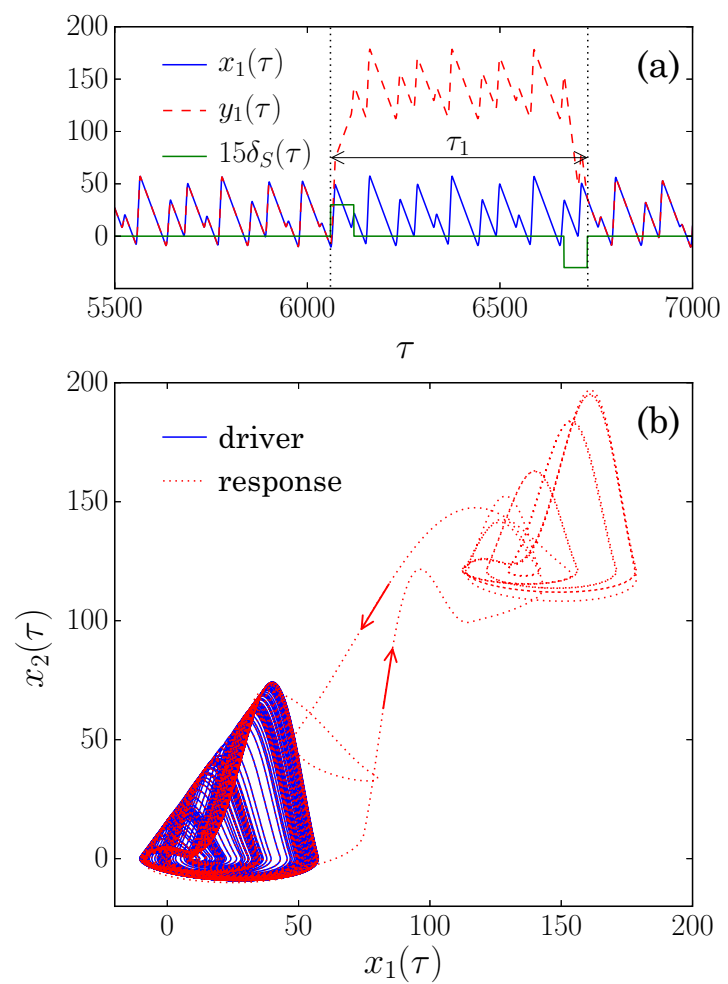

FIG. 4. Effect of two opposite impulses on the response system. Initial conditions are assumed identical, $x_{1}(0)=y_{1}(0)=0$ and hence it shows that the systems are not separated before the application of an impulse.

derivative of the Lyapunov function becomes

$$
\dot{V}_{1} \leqslant \frac{e_{1}^{2}}{4 \gamma}+\delta(\tau)\left|e_{1}\right| .
$$

The response system obviously receives sufficient energy to move away from the initial distance (region I) or to the final saturation distance (region III) asymptotically as we increase the energy $E_{\text {imp }}$ by increasing $\mu$. However, if $x_{1}(0)>0$, $e_{1}(0)>0$ the time derivative of the Lyapunov function in Eq. (10) is given by

$$
\dot{V}_{1} \leqslant\left|\frac{e_{1}^{2}}{4 \gamma}-\delta(\tau) e_{1}\right| .
$$

Therefore, at a critical value $\mu=\mu_{C}=\frac{e_{1}}{4 \gamma h}$, the Lyapunov function follows the relation $\dot{V} \simeq 0$.

Next, we investigate what happens to the leader-follower dynamics when a second impulse negative but equal in magnitude and width to the first impulse is applied to the response. It appears that the second impulse cancels out the effect of the first. The drive and response systems coincide again when the injected energy is removed by the application of a negative impulse as seen in Fig. 4. Figure 4(a) shows the time series of the drive and the response systems, $x_{1}$ and $y_{1}$, respectively. Figure 4(b) shows that the response attractor, after a departure to a targeted distance, returns to the original position and merges with the driver attractor. Arrows show the direction of movements. 


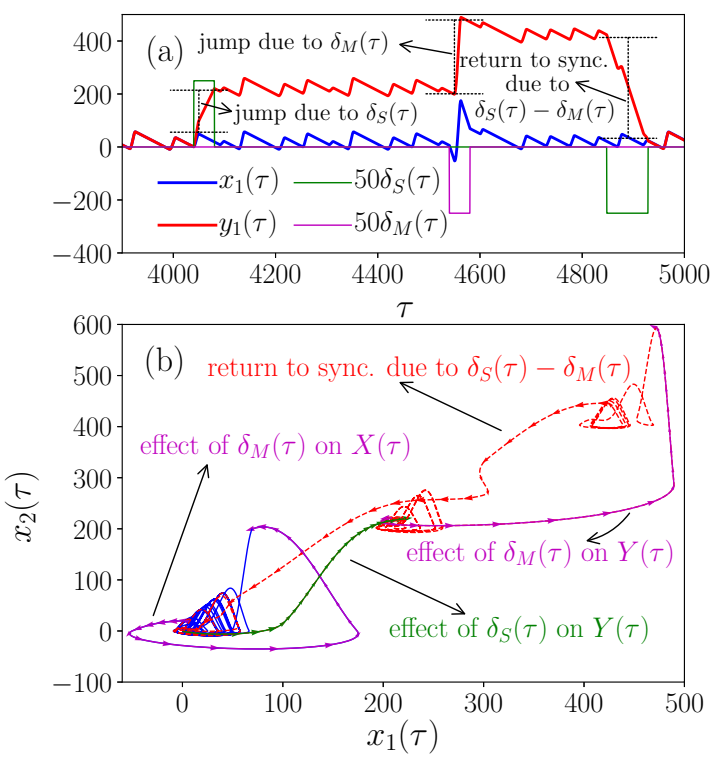

FIG. 5. Impact of two delayed pulses $\delta_{i}(\tau), i=S, M$ on the systems with $\epsilon_{1}=-1$ and $x_{1}(0)=y_{1}(0)=0$. Panel (a) shows the time histories of the signals $x_{1}(\tau)$ (blue solid), $y_{1}(\tau)$ (red solid line) and the impulses $\delta_{S}(\tau)$ (green solid line) and $\delta_{M}(\tau)$ (violet solid line). The first impulse from $\delta_{S}(\tau)$ produces the first jump of the response while the second is the consequence of the negative $\delta_{M}(\tau)$. The synchronization is finally reached due to a negative impulse which respects the relation $\delta_{S}(\tau)-\delta_{M}(\tau)$. Panel (b) presents the same behavior in the plane $\left(x_{1}(\tau), x_{2}(\tau)\right)$.

\section{B. Displacement of attractors: Multiple impulses}

We now consider the case where both the driver and the response (or slave) systems receive separate impulses one after another. We first inject a positive impulse (green) to the response system and then apply another impulse (magenta), of same height and width but negative, to the driver after a finite delay, as shown in Fig. 5(a). Finally, a third one, a negative impulse (green) is sent to the slave an instant later. The response system is subjected to two successive jumps.

The first jump of the slave system (red line) is provoked by the injection of $\delta_{S}(\tau)$ while the second one is the consequence of the application of $\delta_{M}(\tau)$ to the driver. It is interesting to observe that the driver makes a jump by the direct impulse $\delta_{M}(\tau)$ on it, however, it restores its original position (blue line) after a transient time, while the response oscillator needs a negative impulse equal to the total area of the two inputs $\delta_{S}(\tau)$ and $\delta_{M}(\tau)$ to restore its original distance (zero distance in this particular example case) with the driver as shown in Figs. 5(a) and 5(b). This suggests that whatever be the number of external impulses the oscillators receive, the number of impulses applied to the response system to restore its original position of coincidence, should be equal and opposite to the sum of all the inputs. Figure 5(b) summarizes the whole story in 2D projections of the trajectories showing that both the attractors, the driver (blue) and the response (red), start from a coinciding position. The response attractor jumps to a higher location along the green line (green arrows) due to the first impulse $\delta_{S}(\tau)$, and a second jump reaching another higher level along the magenta line (magenta arrows) for a

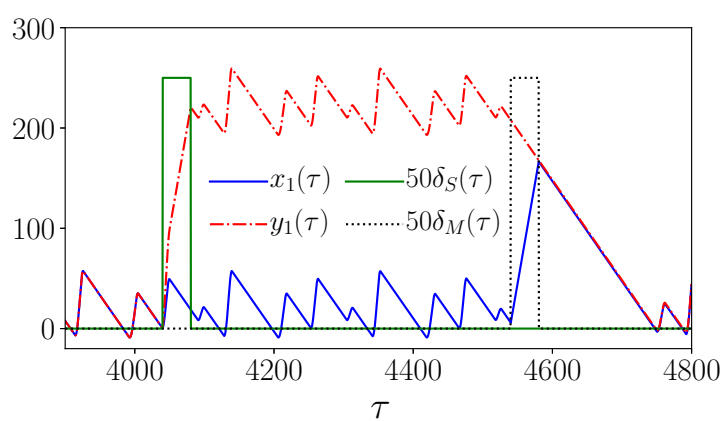

FIG. 6. Impact of two delayed pulses $\delta_{i}(t), i=S, M$ on the systems with $\epsilon_{1}=1$ and $x_{1}(0)=y_{1}(0)=0$.

second negative impulse $\delta_{M}(\tau)$. The response system restores its original position of coincidence with the driver (blue) along the red line (red arrows) when an impulse (green) of size, $\delta_{S}(\tau)-\delta_{M}(\tau)$ is applied. In the meantime, the driver makes an away movement along the magenta line (magenta arrows) when $\delta_{M}(\tau)$ is applied; however, it retrieves its position after a short transient. Alternatively, by applying the second impulse as a positive $\delta_{M}(\tau)$ to the driver, the behavior of both the systems becomes completely different: instead of increasing the distance between the driver and the response systems, the positive impulse $\delta_{M}(\tau)$ leads them to CS and thereby restores their original positions from where they started initially (see Fig. 6).

\section{EXCHANGE OF LEADERSHIP}

As suggested above, the response system can desynchronize and later comes back to rejoin the chorus even after facing external impulses. If we look closely into the details of Fig. 5(a), the leader-follower relation seems to be exchangeable. To understand the meaning of this property of exchanging leader-follower role, let us focus on the time interval $\delta_{M}(\tau)$ [see Fig. 7(a)] or the interval of time between the ends of $\delta_{S}(\tau)$ and $\delta_{M}(\tau)$ [see Fig. 7(b)], namely, $\tau_{2}$. Due to the impulse $\delta_{M}(\tau)$, both systems are desynchronized during the interval of $\delta_{M}(\tau)$, and the length of travel done by the driver is always greater than that of the response system. Second, if we concentrate particularly on Fig. 7(a), we see at the end of the impulse $\delta_{M}(\tau)$ that the response system which is weakly affected by the second impulse $\delta_{M}(\tau)$, seems to have arrived at its permanent position while the driver system is still changing its position [see the break of $x_{1}(\tau)$ at the end of $\delta_{M}(\tau)$ ]. These distinct behaviors between the drive and response systems produce a particular global effect, which is an apparent exchange of leadership: for a short instant of time, the driver seems to become the follower and the response acts as the leader.

This apparent behavior is more pronounced when $\delta_{M}$ is positive. To clarify the relative motion between the driver and the response systems described in Fig. 6, we show their attractors in Fig. 8. The violet line presents the desynchronization track of the response attractor (otherwise red line) along the direction of the violet arrows with the application of $\delta_{S}(\tau)$. The green track with blue arrows indicates the path along which the driver jumps in the duration of $\delta_{M}(\tau)$ while the 

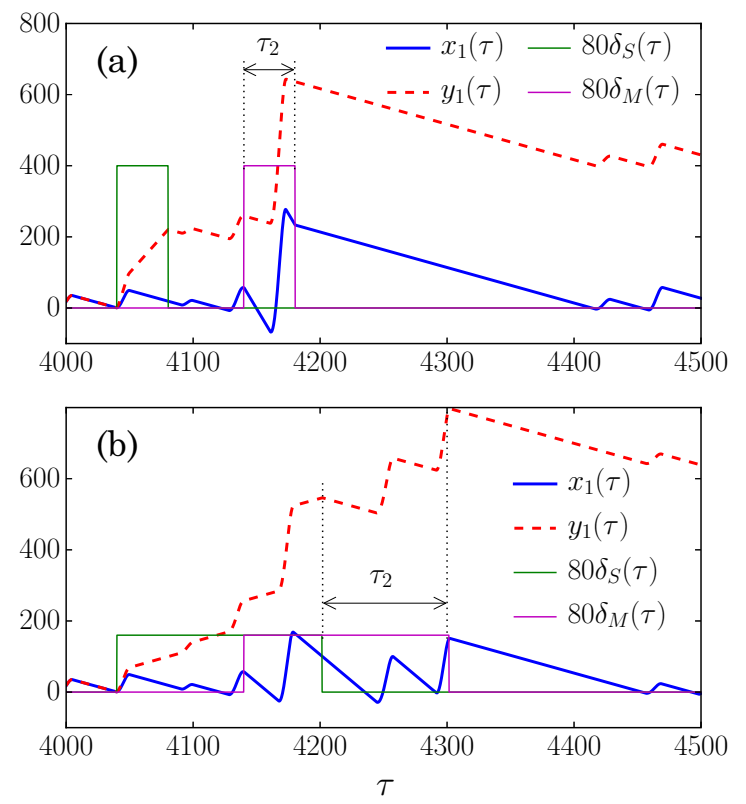

FIG. 7. Exchange of leadership between the master and slave within the $\tau_{2}$ zone, for $\epsilon_{1}=-1$ and $x_{1}(0)=y_{1}(0)=0$. Within the interval of time of application of $\delta_{M}(\tau)$ [see panel (a)] or the interval of time between the ends of $\delta_{S}(\tau)$ and that of $\delta_{M}(\tau)$ [see panel (b)], the distance covered by the driver, dist ${ }_{X}$, and the response, dist ${ }_{Y}$, in the $\tau_{2}$ zone are quite different and dist $_{Y}<$ dist $_{X}$ [in panel (a)],

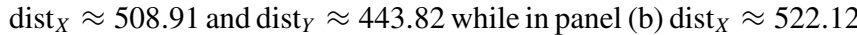
and dist $_{Y} \approx 432.50$.

black track shows how the slave responds to $\delta_{M}(\tau)$. The red arrows indicate the return path of the slave along the black track. Both the master and the slave meet at the end of the black return track of the response. The apparent exchange of leadership clearly appears in the return to synchronization domain where the driver (blue line) adjusts its trajectory to completely synchronize with the response (represented by the red dotted lines).

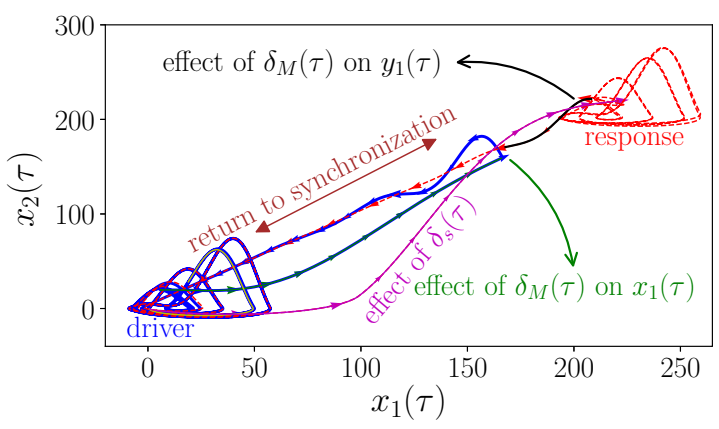

FIG. 8. Global relative motion between both driver and response systems corresponding to Fig. 6. The violet curve presents the getaway of the response with the first kick from $\delta_{S}(\tau)$. The green part shows the jump of the driver during $\delta_{M}(\tau)$ while the black curve shows how the response acts at the same time with $\delta_{M}(\tau)$. At the end of $\delta_{M}(\tau)$ in the return to synchronization region, the response system (red dashed line) appears to be followed by the driver (blue solid line), which adapts itself to synchronize with the response (red dashed line).

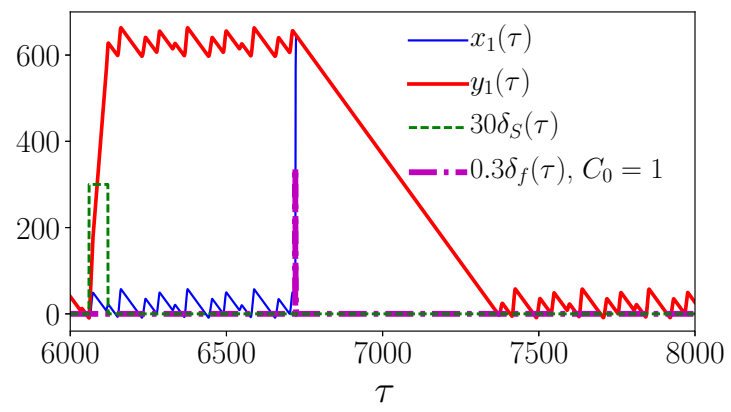

FIG. 9. Response and driver restore synchronization after response sends a short impulse to the leader. Here, the apparent exchange of leadership appears to an observer immediately after the driver jumps to synchronize with the response. Then leader and response move on together in a coherent synchronized way. After the feedback impulse, the driver simply restores its behavior and continues to command synchronization.

We now show the effect of mutual exchange of information between the response and the driver as illustrated in Fig. 9. An impulse (green line) is applied to the response system (red line), which then moves away from the driver (blue line) and departs from their initial position of coincidence for our choice of initial conditions. A feedback from the response system as a small duration pulse (dashed magenta) is then sent, after a while, to the driver, $\delta_{f}=C_{0}\left[y_{1}(\tau)-x_{1}(\tau)\right]$ during a time interval $\left[\tau_{1}, \tau_{1}\left(1+\mu_{f}\right)\right]$. For a suitable value of coupling constant $C_{0}$ and the duration $\mu_{f}$, the driver receives a kick and completely synchronizes with the response when their distance becomes zero. Since the response system remains unperturbed, the dynamics of the driver is only given here after a feedback pulse from the response is applied,

$$
\begin{aligned}
& \dot{x}_{1}=I\left(x_{2}\right)+\delta_{f}(\tau), \quad \dot{x}_{2}=\alpha\left[-x_{3}+I\left(x_{2}\right)\right], \\
& \dot{x}_{3}=\beta\left(-x_{1}+x_{2}-\gamma x_{3}\right),
\end{aligned}
$$

where

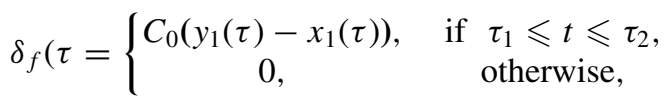

and $\tau_{1}=6720.54, \tau_{2}=6726.6$. Figure 9 shows that the master-slave system regains its original position of coincidence after the short duration feedback signal (dashed-magenta line) is sent to the driver by the response. This tells us that if a response system moves away from the driver by the impact of an external impulse or interceptor, it can send a message in the form of a small duration signal to the driver that suffices to restore their original distance. We can make an analogy of this event with two birds moving in a leader-follower coherent motion. When the follower encounters an obstacle, it moves away from the leader but restores their original positions by sending a feedback message to the leader.

\section{CONCLUSIONS}

The cohesive motion of living and dynamics objects attracted the attention of physicists in the recent past. Some studies were proposed to understand the mechanism of 
such collective motion using both statistical mechanical and dynamical system approaches. To understand the coherent motion of dynamical objects, we propose an interesting toy model of coupled jerk systems in a leader-follower interaction mode where the follower is able to mimic the decision-making process of the driver by copying the nonlinear function of the driver and to send a feedback signal to the leader whenever it is necessary. Using two such dynamical systems, we showed how the trajectories of two dynamical units remain coherent but maintain a safe distance. In the dynamical systems' conventional terms, we called it a special case of GS, which we established both analytically and numerically. The most interesting part of our study was revealed by numerical studies when we found how the response and driver systems reacted to external impulses such as predators and how they avoided the situation by moving away and then coming back to readjust their original distance. The leader-follower role or the master-slave relation was found exchangeable when necessary similar to movements in a flock of birds. We found that the phenomenon is not system specific restricted to these jerk systems only, but generic. We investigated other examples [23-26], the paradigmatic Rössler system, a coupled tunnel diode model, and a Josephson junction-based jerk model. In all of them, we observed coherent motion, but the exchange of leadership appears only for the Josephson junction-based jerk model, apart from the one described in this manuscript (details can be seen in the Appendices A, B, and $C$ ). We determine that the expression of the error dynamics should always have at least one time derivative equal to zero and no nonlinear term for these phenomena to be true. That is why the nonlinearities in the drive and the response systems are the same. We plan to extend our results to a large number of such dynamical units which surprisingly mimic the movement of flocking birds, not presented here. We need more rigorous studies before presenting them in the future.

\section{ACKNOWLEDGMENTS}

P.L., S.K.D., P.M., and H.A.C. thank ICTP-SAIFR and FAPESP Grant No. 2011/11973-4 for partial support. P.L. acknowledges support by the FAPESP Grant No. 2014/13272-1. S.K.D. acknowledges support by the University Grants Commission (India) under the Emeritus Fellowship.

\section{APPENDIX A: RÖSSLER SYSTEMS}

As we mentioned in the Introduction, the response system moves away from the driver and after a short while they start moving in a synchronized state and maintain a constant distance of separation. Eventually, they can restore their original position when a negative pulse is applied or an appropriate feedback pulse is sent to the driver. This coherent dynamics is not restricted to the jerk system only; here we show that this effect appears also in the Rössler and tunnel diodes systems, when we appropriately copy the nonlinearity of the driver into the response system, such that two variables become identical in the driver and response systems, and an appropriately defined Lyapunov function remains bounded in time by a positive constant as shown above for the jerk System.

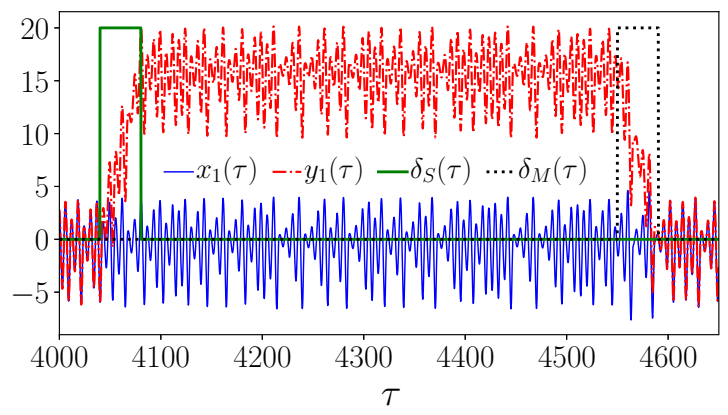

FIG. 10. Rössler systems showing coherent behavior for the initial conditions $(0.3,0.0,-1.0)$ for the driver Eq. (A1) and $(-0.3,0.0,0.1)$ for the response Eq. (A2).

We define the master and follower Rössler systems as given, respectively, by Eqs. (A1) and (A2):

$$
\begin{aligned}
& \dot{x}_{1}=-x_{2}-x_{3}, \\
& \dot{x}_{2}=x_{1}+a x_{2}+\delta_{M}(\tau), \\
& \dot{x}_{3}=b x_{1}+x_{1} x_{3}-c x_{3}, \\
& \dot{y}_{1}=-y_{2}-y_{3}, \\
& \dot{y}_{2}=x_{1}+a x_{2}+\delta_{S}(\tau), \\
& \dot{y}_{3}=b y_{1}+x_{1} x_{3}-c y_{3},
\end{aligned}
$$

with the following parameters: $a=0.36, b=0.4$, and $c=$ 4.5. The results are shown in Fig. 10. The response system (red line) moves away from the driver (blue line) by the impact of a impulse (green) on it, however, rejoins the driver when an impulse (magenta) is applied to the driver at a later instant of time.

\section{APPENDIX B: TUNNEL DIODE SYSTEMS}

We show the similar coherent motion in a tunnel diode system [23] as shown in Fig. 11. The master and response

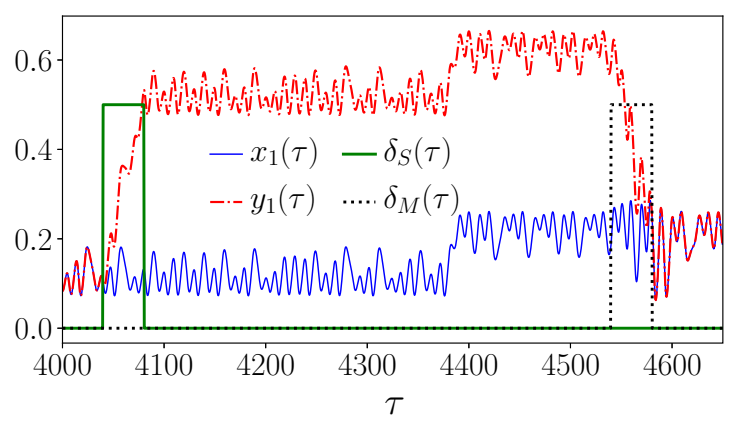

FIG. 11. Tunnel diode based chaotic systems showing coherent behavior for the initial conditions $(0.0,0.27,0.008)$ for the driver Eq. (B1) and $(0.0,0.1,0.1)$ for the response Eq. (B2). 


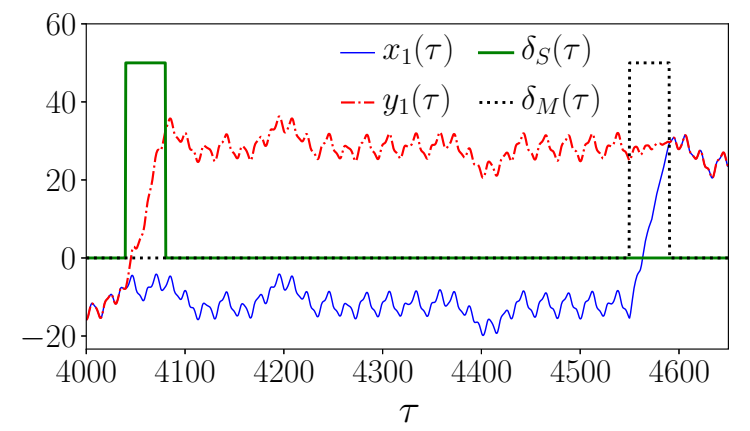

FIG. 12. Josephson junction-based chaotic jerk systems showing coherent behavior for the initial conditions $(0.0,0.4,0.0)$ for the driver Eq. (C1) and $(0.0,-1.0,1.0)$ for the response Eq. (C2). As in Fig. 6, the exchange of leadership is shown by the fact that the driver (blue solid line) is the one that jumps to synchronize with the response system (red solid line) because of the injection of the impulse $\delta_{m}(\tau)$ (violet solid line).

system are

$$
\begin{aligned}
& \dot{x}_{1}=\alpha\left(x_{2}-x_{1}-r f_{n}\left(x_{1}\right)\right)+\delta_{M}(\tau), \\
& \dot{x}_{2}=\beta\left(x_{1}-x_{2}+r x_{3}\right), \\
& \dot{x}_{3}=\gamma\left(E-x_{2}\right), \\
& \dot{y}_{1}=\alpha\left(x_{2}-x_{1}-r f_{n}\left(x_{1}\right)\right)+\delta_{S}(\tau), \\
& \dot{y}_{2}=\beta\left(y_{1}-y_{2}+r y_{3}\right), \\
& \dot{y}_{3}=\gamma\left(E-y_{2}\right),
\end{aligned}
$$

where $f_{n}\left(x_{1}\right)=a_{1}\left(x_{1}-b\right)^{3}-a_{2}\left(x_{1}-b\right)+a_{3}$. For $a_{1}=$ $1.3242872, a_{2}=0.06922314, a_{3}=0.00539, b=0.167, \alpha=$ $2.457222, \quad \beta=0.277778, \gamma=0.225, \quad r=16$, and $E=$ 0.253208 for the driver and the response systems, respectively, both systems are chaotic. The results are shown in Fig. 11.

\section{APPENDIX C: JOSEPHSON JUNCTION-BASED JERK SYSTEM}

We studied the coherent motion in several well-known jerk systems in Refs. [24-26]; however, we restricted ourselves to the Josephson junction-based jerk system studied in Ref. [26],

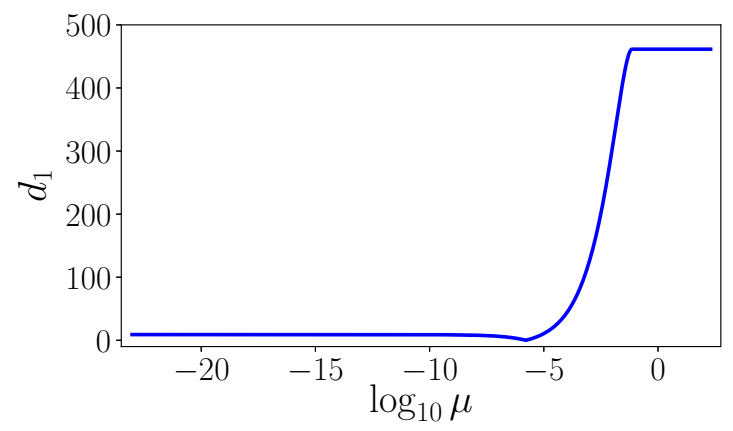

FIG. 13. Separating distance of the drive-response attractors against the width $\mu$ of the external pulse for $x_{1}(0)=9$ and $y_{1}(0)=0$ showing similar result as in Fig. 3(a)

simply because it also presents the exchange of leadership as shown in Fig. 6. The master and response systems are

$$
\begin{aligned}
& \dot{x}_{1}=x_{2}+\delta_{M}(\tau), \\
& \dot{x}_{2}=x_{3}, \\
& \dot{x}_{3}=-a x_{2}-a x_{3}+a \sin \left(2 \pi b x_{1}\right), \\
& \dot{y}_{1}=x_{2}+\delta_{s}(\tau), \\
& \dot{y}_{2}=y_{3}, \\
& \dot{y}_{3}=-a y_{2}-a y_{3}+a \sin \left(2 \pi b x_{1}\right),
\end{aligned}
$$

where $a=0.3$ and $b=0.25$. For the initial conditions $(0.0,0.4,0.0)$ and $(0.0,-1.0,1.0)$, respectively, for the driver and response systems, we obtain the time series shown in Fig. 12. Both the coherent motion between both systems as well as the apparent exchange of leadership when the impulse $\delta_{M}$ is injected, are clearly seen.

In Fig. 13 we present the separating distance between the drive and response attractors versus the width $\mu$ of the external pulse for a selected value of $x_{1}(0)$ and $y_{1}(0)=0$ to show that its particular behavior discussed in Fig. 3 is not restricted to just the systems gave in Eqs. (1) and (2). We found similar results for all the systems presented here in the appendices. In the case of this Josephson junction-based jerk system, we fixed $(9.0,-0.6,-1.0)$ and $(0.0,0.6,0.1)$ the initial conditions for the driver and the response systems, respectively.
[1] A. S. Pikovsky, M. G. Rosenblum, and J. Kurths, Synchronization: A Universal Concept in Nonlinear Science (Cambridge University Press, Cambridge, 2011).

[2] A. Argyris, D. Syvridis, L. Larger, V. Annovazzi-Lodi, P. Colet, I. Fischer, J. Garcia-Ojalvo, C. R. Mirasso, L. Pesquera, and K. A. Shore, Nature (London) 438, 343 (2005).

[3] S. Boccaletti, V. Latora, Y. Moreno, M. Chavez, and D.-U. Hwang, Phys. Rep. 424, 175 (2006).

[4] L. M. Pecora and T. L. Carroll, Phys. Rev. Lett. 64, 821 (1990).

[5] J. Kurths, Chaos 26, 030401 (2016); J. R. Terry, K. S. Thornburg, Jr., D. J. DeShazer, G. D. Vanwiggeren, S. Zhu, P. Ashwin, and R. Roy, Phys. Rev. E. 59, 4036 (1999).
[6] R. Lhermerout, N. Bruot, G. M. Cicuta, J. Kotar, and P. Cicuta, New J. Phys. 14, 105023 (2012).

[7] A. K. Engel, P. Konig, A. K. Kreiter, and W. Singer, Science 252, 1177 (1991).

[8] D. J. Watts and S. H. Strogatz, Nature (London) 393, 440 (1998); S. H. Strogatz, ibid. 410, 268 (2001).

[9] R. Albert and A.-L. Barabási, Rev. Mod. Phys. 74, 47 (2002).

[10] M. E. J. Newman, SIAM Rev. 45, 167 (2003).

[11] A. Attanasi, A. Cavagna, L. D. Castello, I. Giardina, T. S. Grigera, A. Jelić, S. Melillo, L. Parisi, O. Pohl, and E. Shen, and M. Viale, Nat. Phys. 10, 691 (2014). 
[12] W. Bialek, A. Cavagna, I. Giardinab, T. Morad, E. Silvestri, M. Vialeb, and A. M. Walczake, Proc. Natl. Acad. Sci. USA 109, 4786 (2012).

[13] A. Cavagna, L. D. Castello, I. Giardina, T. Grigera, A. Jelic, S. Melillo, T. Mora, L. Parisi, E. Silvestri, M. Viale, and A. M. Walczak, J. Stat. Phys. 158, 601 (2015).

[14] T. Vicsek and A. Zafeiris, Phys. Rep. 517, 71 (2012); K. Ozogány and T. Vicsek, J. Stat. Phys. 158, 628 (2015) and refs. therein.

[15] O. Petit and R. Bon, Behav. Process 84, 635 (2010).

[16] M. Nagy, Z. Ákos, D. Biro, and T. Vicsek, Nature (London) 464, 890 (2010).

[17] H. D. I. Abarbanel, N. F. Rulkov, and M. M. Sushchik, Phys. Rev. E 53, 4528 (1996).

[18] E. Padmanaban, C. R. Hens, and S. K. Dana, Chaos 21, 013110 (2011); M. Vigneshwaran, S. K. Dana, and E. Padmanaban, Euro. Phys. Lett. 116, 50010 (2016); P. K. Roy, C. Hens, I.
Grosu, and S. K. Dana, Chaos 21, 013106 (2011) and refs. therein.

[19] R. Tchitnga, Tekou Nguazon, P. H. Louodop Fotso, and J. A. C. Gallas, IEEE Trans. Circ. Syst-II: Express Briefs 63, 239 (2016).

[20] F. M. M. Kakmeni, S. Bowong, D. V. Senthilkumar, and J. Kurths, Chaos 20, 043121 (2010).

[21] M. Sekieta and T. Kapitaniak, Int. J. Bifur. Chaos 6, 1901 (1996).

[22] R. Femat and G. Solís-Perales, Phys. Rev. E 65, 036226 (2002).

[23] P. Louodop, H. Fotsin, M. Kountchou, Elie B. Megam Ngouonkadi, H. A. Cerdeira and S. Bowong, Phys. Rev. E 89, 032921 (2014).

[24] J. C. Sprott, IEEE Trans. Circuits Systems II: Express Briefs 58(4), 240 (2011).

[25] K. Sun and J. C. Sprott, Int. J. Nonlin. Sci. Numer. Simul. 10, 1443 (2009).

[26] M. E. Yalcin, Chaos, Solitons Fractals 34, 1659 (2007). 